\title{
INVESTIGACIONES
}

\section{Aperturas y cierres para la inclusión educativa de niños/as migrantes en Chile}

\author{
Facilitators and barriers for the educational inclusion \\ of migrant children in Chile
}

\author{
Rolando Poblete Melis ${ }^{a}$, Caterine Galaz Valderrama ${ }^{b}$ \\ a Departamento de Investigación Educativa, Facultad de Ciencias de la Educación, Universidad Central. \\ Correo electrónico: rpobletem@ucentral.cl \\ ${ }^{\mathrm{b}}$ Escuela de Trabajo Social, Facultad de Ciencias Sociales, Universidad de Chile. \\ Correo electrónico: cgalazvalderrama@uchile.cl
}

\begin{abstract}
RESUMEN
El aumento de población extranjera demandando servicios educativos en Chile ha requerido abordar aspectos políticos (decretos y normativas) que permitan su acceso al sistema educacional; pero también las escuelas han debido generar estrategias para avanzar hacia la inclusión. La investigación que da origen a este artículo tuvo como objetivo conocer, a través de una metodología cualitativa, las trayectorias de inclusión educativa de niños y niñas migrantes en cuatro escuelas y un liceo de la ciudad de Santiago, identificando los factores que favorecen y dificultan el proceso de inclusión, a nivel del acceso al sistema educativo y en las relaciones que se dan en su interior. Entre los principales resultados aparecen los problemas derivados de la condición de irregularidad migratoria, lo que afecta el acceso a los beneficios del sistema educacional, además de tensiones en las relaciones entre estudiantes chilenos y extranjeros que pueden dar pie a situaciones de bullying.
\end{abstract}

Palabras clave: migración, derecho a la educación, escolarización, acceso, discriminación

\section{ABSTRACT}

The increase in the foreign population demanding education services in Chile has made it necessary to address political issues to enable their access to the educational system; however, schools have also had to develop strategies to attain inclusion. The aim of the research behind this article was to discover, through a qualitative methodology, the trajectory towards the educational inclusion of migrant boys and girls that took place in four elementary and one high school in the city of Santiago, identifying the factors that favor and hinder this process, including their access to the educational system and the relations that are established within schools. Some of the main discoveries of this research include problems related to the condition of irregular migration -which affects access to the benefits of the educational system- and also tensions in the relations between Chilean and foreign students, which can lead to situations of bullying.

Key words: migration, right to education, schooling, access, discrimination 


\section{ANTECEDENTES}

No cabe duda que Chile se ha transformado en polo de atracción para diversos colectivos migrantes provenientes en su mayoría de los países de la región, lo que se ha traducido en el incremento del número de personas nacidas en el extranjero que se han asentado en el país. Así lo muestran los distintos estudios (Martínez, 2003; Stefoni, 2003; Autores, 2015) y bases de datos existentes al respecto (CASEN 2013, 2015; DEM, 2015). Aunque dependiendo de la fuente consultada los datos tienden a variar (Stefoni, 2003), es posible estimar que los y las migrantes representan entre un 2,5\% y un 3,0\% del total de población nacional, muy por debajo todavía de países con similar nivel de desarrollo como Argentina, cuya población migrante supera el 4\% (Martínez, 2003).

La mayor parte de la población extranjera en el país se encuentra en edad económicamente activa. Sin embargo, con el inicio del proceso de reunificación familiar a partir del año 2000 (Pavez, 2012) ha comenzado a llegar población infantil, estimándose que el 19\% del total de extranjeros se encuentra en el rango etario 0 a 19 años (DEM, 2015), transformándose en potenciales demandantes de servicios educativos en las regiones y territorios en los que se han asentado junto a sus familias, en particular en la región metropolitana que concentra casi el 67\% de los y las migrantes, seguido por la región de Valparaíso y Tarapacá en el norte del país, con 4,7\% y 4,0\% respectivamente (CASEN, 2013). Y dentro de la región metropolitana, la mayor parte de ellos/as se ubica en las comunas de Santiago, Independencia, Recoleta y Estación Central.

De acuerdo a datos del Ministerio de Educación de Chile de los cerca de 3.550.000 niños y niñas matriculados/as en el sistema educativo nacional al año 2015, 30.625 son extranjeros/as, lo que representa menos del $1 \%$ de la población escolar. La mayor parte son peruanos/as, seguidos por colombiano/as, concentrados fundamentalmente, y al igual que la población adulta, en la región metropolitana de Chile. Respecto de la distribución por nivel educacional, el 13\% se encuentra matriculado en educación prebásica; el $68 \%$ en básica y el 19\% en secundaria (Mineduc, 2015).

Pese a que el número de niños y niñas extranjeras matriculados en el sistema educativo aún es bajo, su presencia y mayor visibilidad ha supuesto un desafío importante para el Estado, la propia sociedad y las escuelas. En efecto, la población migrante que demanda servicios educativos ha requerido abordar aspectos de carácter político, asociados a normativas y decretos que permitan garantizar su acceso al sistema educacional, lo que es coherente con las convenciones internacionales que Chile ha ratificado y que consagran tal derecho ${ }^{1}$.

Estas convenciones han permitido poner como centro de la acción del Estado la perspectiva de los derechos humanos en temas migratorios, lo que termina generando una discusión, en definitiva, respecto de la ampliación de los márgenes de la ciudadanía actual (Subirats, 2010).

Pero no solo el Estado ha respondido con normativas con este nuevo escenario, también han sido las propias escuelas las que han debido diseñar estrategias para avanzar hacia la inclusión y lograr que niños y niñas accedan al aprendizaje en un marco de respeto a la diversidad que representan.

Las principales convenciones que garantizan el derecho a la educación para la población migrantes son: Convención Internacional sobre la Protección de los Derechos de Todos los Trabajadores Migratorios y sus Familias; Convención sobre los Estatutos de los Refugiados; Convención Internacionales sobre la eliminación de todas las formas de discriminación racial; Convención Internacional sobre los derechos del niño. 
Desde el año 2005 existe una normativa del Ministerio de Educación que permite la matrícula en el sistema educativo de niños y niñas migrantes al margen de la situación migratoria de sus familias (Mineduc, 2005), sin embargo, pese a este decreto se ha puesto en evidencia que ya en el acceso a los centros educativos se producen barreras significativas que dificultan los procesos de inclusión, especialmente para quienes están en situación irregular, tales como contar solo con una matrícula provisoria; dificultades para participar de los beneficios que derivan de su condición de estudiantes (becas de alimentos; subvención escolar preferencial) o incluso imposibilidad de certificar los estudios realizados (Autor et al., 2015). A partir del año 2017 ha entrado en vigencia una nueva normativa que debiera resolver estos problemas y que en lo sustantivo dice que el Ministerio de Educación entregará a través de las oficinas de atención ciudadana un identificador provisorio escolar (PIE) que operará para todos los efectos como un rut o número de identidad temporal (Mineduc, 2016).

Lo anterior ratifica que de parte del Estado se han generado acciones para lograr el acceso de los migrantes a los servicios sociales, sin embargo, no han tenido necesariamente los resultados esperados, sobre todo a nivel local de los servicios (Martínez, 2011).

En cuanto a lo que ocurre al interior de los centros educativos, algunas investigaciones (UNICEF 2004, 2011) indican que los/as estudiantes chilenos/as tienen niveles de prejuicio preocupantes hacia niños y niñas de otras nacionalidades, considerando incluso como inferiores a quienes provienen de los países vecinos, especialmente de Perú y Bolivia. De hecho existen evidencias que indican que "la experiencia migratoria de las niñas y niños en Chile está fuertemente marcada por las constantes situaciones de discriminación y racismo de las cuales son víctimas" (Pavez, 2012: 87). El color de la piel, la forma de hablar y otras características distintivas se constituyen en elementos que generan marginación por parte de niños y niñas chilenas.

Estos antecedentes hacer ver que los y las estudiantes migrantes enfrentan una serie de dificultades para acceder, insertarse y progresar en el sistema escolar, de ahí que la pregunta por las trayectorias de inclusión educativa es especialmente importante, abarcando múltiples posibilidades de respuesta.

En ese marco, este artículo da cuenta de los resultados de una investigación en torno a la presencia en el sistema educativo chileno de estudiantes extranjeros. En concreto, el objetivo del estudio ha sido conocer las trayectorias de inclusión educativa de niños y niñas migrantes en cuatro escuelas y un liceo de la ciudad de Santiago, identificando los factores que favorecen y dificultan el proceso de inclusión, en particular a nivel del acceso al sistema educativo y en cuanto a las relaciones que se dan al interior de los establecimientos.

\section{CONSIDERACIONES TEÓRICAS}

En el año 2015 se promulgó en Chile la ley de inclusión educativa, de aplicación gradual en el tiempo, que entre otras cosas establece como principio la no discriminación arbitraria, lo que supone el rol del Estado a favor de la inclusión e integración en todos los establecimientos del país, así como la promoción de la dignidad del ser humano (MINEDUC, 2015).

Es relevante tener a la vista esta normativa porque las escuelas con presencia de estudiantes diversos en su interior (y la interacción entre éstos), ofrecen numerosos hechos de interés en especial en cuanto a los procesos de incorporación, adaptación y progreso que viven los grupos minoritarios en los centros educativos (Ogbu, 1991; Carrasco, 1998; 
Autores, 2007) y que van desde el momento en que acceden al espacio escolar hasta que egresan de éste, constituyendo una trayectoria de inclusión (o exclusión) educativa (Booth y Ainscow, 2000).

Un primer punto a tener en cuenta para pensar la inclusión educativa es el rol de los funcionarios de primera línea, vale decir, quienes reciben en los espacios de atención social a las familias migrantes o los propios niños determinando sus posibilidades de acceso a los servicios educacionales. Las investigaciones realizadas al respecto han mostrado que los y las funcionarios cumplen por una parte un rol definido administrativamente, pero por otra ponen en juego sus propias características personales en el ejercicio de ese rol, operando como facilitadores u obstaculizadores del acceso a los servicios del Estado de las familias migrantes (Lipsky 1980, 1996; Dubois, 2003).

En esa misma línea -aunque referido a un ámbito mas general y no limitado solo a las escuelas-, Torres y Garcés (2013) muestran que el acceso a los servicios públicos por parte de los migrantes se encuentra atrapado en las opiniones y percepciones que los migrantes y nativos construyen de sí, en relatos sobre la migración, su magnitud, su integración y la escasez de los servicios públicos. Así "el imaginario generado de una relación de competencia por los servicios públicos entre los inmigrantes y los autóctonos determinaría, en alguna manera, la actuación de los funcionarios públicos encargados de brindar los servicios públicos" (Torres y Garcés, 2013: 5).

En segundo término, la inclusión educativa no solo remite a un concepto, sino también a determinadas prácticas destinadas a favorecer la participación de todos y todas en los procesos educativos (Blanco, 1999; Booth y Ainscow, 2000). En ese sentido, la inclusión sostiene una perspectiva basada en los derechos humanos, en la cual todos pueden ejercer el derecho a la educación, al margen de cualquier consideración relacionada con la etnia, género, estilos de aprendizaje o necesidades educativas especiales (UNESCO, 2005).

Echeita (2008) por su parte señala que la inclusión educativa refiere a un valor, a una aspiración en la cual todos y todas deben ser considerados de la misma forma. Sin embargo, no se puede desconocer que existen grupos que por sus características presentan mayores riesgos de vivir en forma efectiva ese ideal de inclusión. Es lo que ocurre, por ejemplo, con niños y niñas con necesidades educativas especiales; quienes presentan alguna discapacidad; los hijos e hijas de migrantes o quienes no conocen la lengua del país en el cual se han asentado debido a procesos de movilidad. Sin embargo, no se puede considerar solo como un ideal, dado que supone componentes prácticos como el hecho de aspirar a "un aprendizaje y un rendimiento escolar de calidad y exigente con la capacidad de cada estudiante" (Echeita, 2008: 11).

En esos términos la inclusión educativa plantea analizar las políticas, las prácticas y las culturas para eliminar las barreras que impiden el acceso y participación de todos en el sistema educativo (Booth y Ainscow, 2000), lo que por cierto implica cambios en las metodologías docentes, adaptaciones curriculares, revisión de las políticas, trabas administrativas, etc. Más concretamente, entre otras cosas la inclusión educativa (Booth y Ainscow, 2000):

1. Implica reestructurar la cultura, las políticas y las prácticas de los centros educativos para que puedan atender a la diversidad del alumnado.

2. Refiere al refuerzo mutuo de las relaciones entre los centros escolares y sus comunidades. 
3. Implica que todos/as los sujetos de una determinada comunidad aprendan juntos, en forma independiente a sus condiciones personas, sociales o culturales, incluidos aquellos que presentan una discapacidad.

4. No exige requisitos de entrada ni mecanismos de selección o discriminación de ningún tipo para hacer realmente efectivos los derechos a la educación, a la igualdad de oportunidades y a la participación.

Como se desprende de lo señalado, son varios los campos sobre los cuales se debe intervenir, de forma tal que lo que hace "una escuela plural y democrática es abrirse institucional, organizativa y curricularmente hacia las diversas formas culturales y de socialización que caracterizan a sus estudiantes, docentes y el entorno donde se ubica" (Autor, 2009:186). Dicho de otra forma, la acción de la escuela puede ser aprovechada para lograr la valoración de las manifestaciones culturales propias de sociedades multiculturales como las nuestras y "puede jugar un papel significativo en la erradicación del racismo y de otras formas de enfrentamiento interhumano (esto es la mentalidad del nosotros versus ellos), haciendo un esfuerzo consciente para exponer las bases de los mitos que rodean y justifican la superioridad de unos grupos humanos sobre otros" (García y Sáez, 1998: 31). En ese sentido, es fundamental repensar las funciones de cohesión social, integración política y apoyo al desarrollo personal de las escuelas (Fernández y Terrén, 2008).

En esa línea y siguiendo a Chiodi y Bahamondes (2001), los procesos educativos en sociedades diversas debieran afrontar tres grandes desafíos: i) los derechos de grupos étnicos, culturales o minorías nacionales; ii) la lucha contra el prejuicio racial y la búsqueda de formas de integración interétnica no asimilacionista en los países donde se han asentado definitivamente masas de origen foráneo; iii) la adecuación de los sistemas educativos y de la pedagogía a las exigencias de un mundo cada vez más transnacionalizado.

Esto adquiere pleno sentido si se considera que las relaciones entre grupos diversos en el espacio escolar tienden a darse muchas veces desde el conflicto (Ogbu, 1991; López, 1997; Leiva, 2008; Fernández y Terrén, 2008; Autor, 2009). En efecto, debido a los crecientes procesos de diferenciación social toda la institución escolar debe enfrentarse con alumnos étnica y culturalmente diversos. Sin embargo, pueden surgir conflictos cuando la sociedad y la escuela desconocen tal hecho ordenándose desde una perspectiva normalizadora y excluyente (Autores, 2007). Para muchos grupos culturales y sociales llegar a la escuela constituye un verdadero choque; ésta al no tener en cuenta los mecanismos de socialización por los cuales ha transitado la vida de sus estudiantes (su historia), está negando una parte importante y llena de vitalidad de éstos (Autores, 2007).

Analizar entonces las relaciones adquiere relevancia en el marco de los procesos de inclusión educativa, más si se considera que las evidencias existentes en Chile han mostrado que muchas veces la experiencia educativa de niños y niñas migrantes está determinada por situaciones de racismo y discriminación por parte de sus compañeros chilenos (UNICEF 2004, 2011; Pavez, 2012).

En definitiva, promover la inclusión educativa implica generar nuevas políticas, culturas y prácticas (Booth y Ainscow, 2000). Lo primero refiere al conjunto de normas e instrumentos que regulan u organizan los sistemas educativos, tanto a nivel general como en las propias escuelas. Lo segundo implica reconocer los valores existentes a nivel de los centros educativos y los mecanismos de participación que aseguran el acceso de todos y todas tanto al currículum como a las distintas acciones educativas. Finalmente, las prácticas 
hacen referencia a la forma en que la escuela y sus diferentes actores reflejan tanto las políticas como las culturas inclusivas, para lograr la integración de la enseñanza y los apoyos para alcanzar el aprendizaje y superar las barreras que lo limitan (Booth y Ainscow, 2000).

\section{CONSIDERACIONES METODOLÓGICAS}

Este estudio fue realizado bajo un enfoque de tipo cualitativo-hermenéutico, lo que implica que fue la perspectiva de los actores el eje tanto para el proceso de generación de información, como para su análisis e interpretación. En tal sentido, los métodos y técnicas cualitativas apuntan a la comprensión de fenómenos en relación a los significados que los sujetos otorgan a su realidad (Ruiz Olabuenaga, 2012). Ante ello, se priorizaron como técnicas la entrevista en profundidad (20 entrevistas) y grupos de discusión de niñas/os migrantes y nacionales (10 en total). En forma adicional se trabajó con fuentes secundarias, especialmente documentos oficiales, decretos, normativas, bases de datos y otros. La investigación se llevó a cabo en cuatro comunas de la región metropolitana de Chile: Santiago, Recoleta, Independencia y Quilicura. Las tres primeras comunas concentran la mayor cantidad de población extranjera en el país, aunque se optó por incluir Quilicura debido a la significativa presencia de población haitiana, lo que ha supuesto desafíos mayores en los espacios educativos debido a la diferencia idiomática. En cada una de estas comunas se escogió a aquella escuela con mayor presencia de niños y niñas extranjeras, y en la comuna de Santiago también se incorporó un liceo con el fin de conocer las trayectorias de inclusión más allá de la enseñanza básica.

Los agentes seleccionados para participar en el estudio fueron directivos; docentes y estudiantes. En el análisis que se presenta se abordaron dos dimensiones: el nivel de acceso al sistema y los tipos de relaciones sociales que se evidencian en las escuelas. Estas dimensiones fueron "leídas" con el fin de identificar los factores que desde ellas facilitan o dificultan el proceso de inclusión de niños y niñas migrantes en los centros educativos que fueron considerados. Finalmente, se incorporan algunos relatos que emergieron desde las entrevistas con el fin de otorgar mayor fuerza a las ideas expresadas.

\section{RESULTADOS}

\subsection{FACILITADORES Y DIFICULTADES EN EL ACCESO AL SISTEMA EDUCATIVO}

De acuerdo a los datos extraídos de la investigación, se destaca como facilitador para la inclusión el mismo marco general que regula el acceso de la población infantil de origen migrante. Se recalca que el conjunto de decretos que norman y regulan el acceso de niños/ as migrados/as a las escuelas públicas del país, resguarda a nivel legal el derecho a la educación independiente de su nacionalidad. Esto daría cuenta de que, desde el aparato estatal, existe un primer paso a nivel de "reconocimiento social" de que los niños/as extranjeros/as requieren tener igualdad de acceso a nivel educativo:

“Antes era todo más complejo... aunque también habían pocos niños migrantes, pero después desde el ministerio se facilitó el acceso y el papeleo que tenían que 
hacer...es que antes era todo muy engorroso y costoso también, pero ahora no es así, y entonces a la gente no le cuesta matricular a los niños en las escuelas públicas, en algunas sobre todo como la nuestra, pero también se debe a que cambió la normativa de arriba y garantizó que esto fuera más expedito" (Entrevista Docente).

Este reconocimiento legal además implica que las escuelas garanticen este derecho incluso independiente de la condición migratoria de sus progenitores. Este piso de entrada, como señalan algunas fuentes entrevistadas, ha abierto la vía de regularización de todo el grupo familiar, lo que ha redundado en posibilitar mejores oportunidades sociales para esos núcleos:

"Sí, las escuelas con apoyo del municipio, de la oficina, podemos hacer todo el trámite para legalizar a la familia completa, y ya sabemos qué hacer y cómo, qué papeles llevar y rápidamente las personas pueden arreglar su situación... el problema está en la poca gente que entra al país por donde no se debe, y queda con problemas de legalidad... son los menos eso sí. Hemos tenido casos, pero finalmente esos niños terminan devolviéndose a sus países porque no hay forma de que la familia pueda encontrar una solución" (Entrevista Docente).

De esta manera, las escuelas comienzan a tener un rol articulador de problemáticas que viven las familias migrantes con su instalación en la comunidad. En el fondo, la experiencia de algunas escuelas analizadas muestran como su acción es una herramienta efectiva de inclusión social no sólo de sus estudiantes sino de las familias: las escuelas son los referentes para estos colectivos y un canal efectivo de participación (se destaca en algunas de las escuelas la participación asidua de madres y padres migrados/as). Podemos señalar que la comunidad educativa se está transformando en una herramienta de diagnóstico y de orientación migratoria y de instalación en el nuevo contexto para esos colectivos.

Otro de los facilitadores tiene que ver con la voluntad de los cuerpos directivos de las escuelas, e incluso de la disposición política respecto de la llegada migratoria de algunos alcaldes y alcaldesas municipales, toda vez que las escuelas son de dependencia municipal.

"De todas maneras es una política educativa comunal, pero nosotros ya lo veníamos practicando del año pasado, para nosotros era un rollo, porque finalmente nosotros, mira, los niños no tienen RUT y finalmente no tienen acceso a salud, no tienen acceso a nada y eso es una vulneración de derechos. Entonces la escuela necesariamente debe hacerse parte de ese proceso y a partir de este año se asume como política educativa comunal" (Entrevista Directivo).

Esto se traduce en una disposición de acogida efectiva de los colectivos migrantes en ciertos territorios, y al interior de las escuelas, en el desarrollo de actividades que favorezcan la inclusión de los/as niños/as migrados/as.

"Aquí (en la comuna) nos ha posibilitado continuar, seguir trabajando por la comunidad... porque la gente se estaba yendo a otros establecimientos y los migrantes están entusiasmado en tener esta escuela y lo transmiten así a otras personas para que traigan a sus hijos" (Entrevista Docente). 
Asimismo, la misma presencia de los/as migrantes es un facilitador en sí, no sólo porque la idea de que la presencia de expresiones diversas enriquece, sino también porque su llegada a los establecimientos municipales ha incrementado la matrícula y asegurado de esa manera la sostenibilidad de esa oferta formativa. De hecho, algunas escuelas han podido dar continuidad a estos proyectos ante la baja continua de la matrícula dentro del ámbito público.

Pese a este factor positivo, sólo en algunas escuelas exploradas se desarrolla una planificación a largo plazo que implique una perspectiva intercultural más acabada. En la mayoría, las actividades y actuaciones de mediano y largo plazo, dependen de la voluntad de sus autoridades. En ese sentido, algunas escuelas han desarrollado un proceso de planificación educativa que implica una adecuación no sólo curricular sino del proyecto educativo que integra la variable intercultural. El hecho que muchas de las iniciativas interculturales que se desarrollan requieran del apoyo externo (particularmente del Ministerio de Educación, de los municipios o de entidades no gubernamentales) hace que tengan un carácter esporádico. Uno de los límites más señalados es no poseer recursos especiales a los establecimientos que posibiliten programas más integrados a largo plazo, o la creación de programas de capacitación para sus docentes.

También, en algunas escuelas consultadas se han propuesto planes tendientes a la inclusión, con más o menos participación de las familias y los/as estudiantes. Algunas de las escuelas con más experiencia en la acogida de población inmigrada han buscado generar un cambio cultural educativo donde se asegure ciertos niveles participativos de la comunidad.

Por otro lado, otro facilitador es la proximidad de las escuelas a los entornos inmediatos de la comunidad inmigrada. El análisis permite ver no sólo que las escuelas agrupan una mayor cantidad de niños y niñas migrantes por su espacio de residencia, sino que este hecho según las fuentes es bien valorado por las familias por varios motivos: a) porque facilita el hecho del traslado y lo hace más económico, b) porque las familias migrantes suelen tener jornadas extensas laborales y el hecho de contar con espacios educativos cercanos facilita la gestión familiar de los tiempos.

La concentración educativa en algunos centros, no obstante, no sólo obedece a la ubicación residencial de las personas, sino a variables de pertinencia cultural: esto quiere decir que las escuelas desarrollan planes de acogida abiertos a la población migrante y, por tanto, a través de sus propias redes informales dan a conocer la apertura y disponibilidad a recibir población extranjera, lo que hace que esos establecimientos tengan una mayor cantidad de niñas y niños migrantes. La "fama positiva" de algunos establecimientos en cuanto a su trato y atención a la comunidad inmigrada son factores reconocidos como facilitadores para las familias migrantes.

"Es por el entorno, no sé a qué se debe, pero aquí hay más cantidad de viviendas disponibles para el valor que ellos podrían pagar por arriendo o por compra. Otros barrios ya están consolidados y hay pocos movimientos de compra y venta de viviendas o de arriendo y aquí no. Aquí hay construcciones y muchos departamentos en arriendo. Es muy flotante la población de este sector, por las características del sector. Hay una población fija y una que se quiere ir del sector, que a su vez arrienda acá cuando se va, porque es un barrio que tiene cierto estigma" (Entrevista Directivo). 
Entre las dificultades que se enuncian en las escuelas, se considera el hecho que muchos/as de sus estudiantes carecen de la documentación educativa del país de origen, lo que supone una serie de trabas administrativas en este nuevo contexto. Esto se debe a la imposibilidad de traerlos desde origen y a los costos que implica requerirlos desde Chile. De esta manera, los obstáculos que encuentran algunas escuelas para la inscripción de niños/as extranjeros/as, dicen relación con la necesidad de contar con documentación válida frente al Ministerio de Educación para la matrícula. Estos trámites de acreditación y de certificaciones son realizados por las mismas autoridades de las escuelas, quedando a "su buena voluntad" la búsqueda y presentación de tales documentos. En la mayoría de las escuelas se destaca como buena práctica institucional las formas alternativas para salvar este escollo, sin embargo, se advierte que en relación a otras escuelas se convierte en un problema que resolver cuando podría facilitarse desde una instancia superior estos requerimientos. Por otro lado, se enfatiza que el hecho de favorecer vías alternativas y positivas de ingreso de niños/as migrados/as afectados/as por la falta de documentación, hace que otras escuelas terminen derivándoles estudiantes para que puedan ser matriculados.

Esto lleva, sin duda, a que las escuelas de este estudio evidencien una alta concentración de niños/as migrantes, lo que se suma a la concentración de población extranjera residente en esos territorios. Aunque no cabe duda que esto es positivo, también contribuye a una suerte de "guetificación" ya que se transforman en "escuelas para migrantes", impidiendo la necesaria relación con niños y niñas chilenos/as, lo cual finalmente afecta a los procesos de inclusión.

Por otro lado, la ausencia de documentos o la imposibilidad de realizar gestiones para su regularización académica, puede derivar en otra problemática: la falta de reconocimiento de los estudios realizados en las escuelas nacionales, debido a la ausencia de un certificado que acredite que el/la estudiante ha cursado los niveles respectivos y, por tanto, que pueda ocurrir que cursen los años correspondientes sin poder certificarles adecuadamente. Este es un obstaculizador que debe ser resuelto, ya que afecta la permanencia y el progreso de los/as estudiantes migrados/as en el sistema educacional, con lo cual se ponen en riesgo los principios orientadores de las propias normativas emanadas del Ministerio de Educación. Esto es un tema preocupante si se considera la situación de quienes están próximos a egresar de la enseñanza secundaria, toda vez que no pueden certificar sus estudios ni tampoco pueden inscribirse para rendir la prueba de acceso a la universidad.

Otros de los temas que se vincula con lo anterior es lo referido al acceso a los servicios y beneficios que se derivan de la condición de estudiantes, como pueden ser las becas o acceso a otro tipo de programas públicos. En los casos analizados se destacan actuaciones estratégicas para garantizar que todo el alumnado pueda, por ejemplo, acceder a las becas alimenticias, más allá de si cumplen o no con los requisitos establecidos. Así podemos encontrar algunas escuelas donde se decidió que "todos/as o ninguno/a" y, entonces, las becas alimenticias que llegan se repartan a toda la comunidad. Esto precisamente con el afán de evitar diferenciaciones y malas actitudes en el mismo alumnado: diferencias entre personas chilenas/extranjeras o bien, entre migrantes regulares/irregulares.

"Se les da a todos, todos o ninguno. No podemos estar haciendo diferencias entre nosotros, eso es peor...qué dirá el niño que ve que a uno le dan y a otro no... eso genera recelo. Si hay pocas becas, pues se distribuyen" (Entrevista Directivo). 
En esa misma línea también existen dificultades relacionadas con el acceso a la subvención escolar preferencial, que aplica para aquellos estudiantes en condición de vulnerabilidad que asisten a los establecimientos educacionales. Precisamente, carecer de un documento de identidad nacional (RUT) impide que las escuelas reciban los recursos adicionales asociados a esta subvención. También la inexistencia del RUT en los y las estudiantes afecta en diferentes niveles como la participación en igualdad de condiciones en aspectos educativos generales o académicos.

Por otra parte se destaca una necesidad formativa del personal docente y directivo no cubierta pese a que ya la experiencia de algunas escuelas con la comunidad migrada supera los ocho años. Se recalca la necesidad de capacitación en aspectos de pertinencia cultural de acuerdo a las características específicas de la nueva población que deben acoger, pero también de enfoques interculturales en el ámbito educativo (convivencia escolar, curricular, metodologías).

Asimismo, otra dificultad tiene que ver con la preocupación por el sector de la población migrada que no está regularizada debido a que han entrado al territorio por pasos fronterizos no habilitado. Los/las hijos de estas personas difícilmente pueden llegar a tener la certificación de estudios regular de acuerdo van pasando los cursos formativos, ya que no disponen de la certificación de origen. A diferencias de las personas en situación irregular por motivos de trabajo que pueden encontrar salidas a corto o mediano plazo, las personas que entran por pasos fronterizos no habilitados se encuentran como irregulares pero su acto es considerado delito, ante esto la posibilidad de regularizar su situación es más compleja. Por tanto, la certificación para sus hijos/as cuando acaban los ciclos es un verdadero problema para las escuelas. Si bien en los casos analizados se destaca que se acoge a esta población que es minoritaria, muchas veces terminan saliendo del sistema educativo para regresar a sus países. La preocupación también se destaca respecto de la población en situación irregular, porque precisamente son familias que no pueden acceder a gran parte de la oferta pública y pueden estar cruzando especialmente situaciones de vulnerabilidad social y económica que no son atendidas por las barreras administrativas que les supone el estatus de persona no regular.

\subsection{RELACIONES EN LA ESCUELA: DESDE LA COOPERACIÓN AL BULLYING}

Las desavenencias en las relaciones entre iguales (niños/as chilenos/as y niños/as migrados/ as) son explicadas no desde el eje de procedencia sino más bien como "clásicas" por tramo etario (infantil- adolescente) y conductuales.

"De los años en que llevo acá, eso no se da, por ejemplo acá no se da eso de que si el niño es de raza negra lo van a tratar mal. Acá con el tiempo naturalmente se ha dado el sincretismo cultural. Incluso hace unos años atrás cuando recién llegaron los Peruanos, hubo un choque y se fue haciendo un trabajo, y hubieron muchos cambios respecto a la situación de vida, por ejemplo afuera uno ve casas pero dentro de las casas hay muchos niños que viven en piezas, y ese problema se tomó y ha sido integrado dentro de la escuela, se ha hecho trabajo, se ha hecho integración en el sentido, tomando desde porque años atrás hubo problemas entre los apoderados, pero eso por el desconocimiento que había sobre estas personas, por una cosa de choque cultural" (Entrevista Docente). 
Pese a esta visión más optimista y generalizada, sin duda, un punto que ha sido mencionado es la discriminación o bullying por nacionalidad que viven algunos/as estudiantes de origen extranjero. Emergen casos específicos, donde sí consideran que su nacionalidad pesa a la hora de relacionarse con sus pares. Tales conductas se concentran más en personas afrodescendientes.

En los grupos de discusión con niños/as chilenos/as y migrantes emerge la permanencia de prejuicios y estereotipos respecto de la población extranjera por parte de algunos niños y niñas chilenos/as, de parte de algunos profesores y directivos, y de la comunidad donde residen. Aun así, los niños y niñas extranjeros/as destacan que esta percepción negativa es mayor fuera de la escuela que dentro. En ese sentido, a veces la escuela emerge como un espacio socio-simbólico en que las familias migrantes se sienten más acogidas que en otros espacios sociales. Esto lleva a que muchas participen activamente dentro de los centros de padres y madres, y también los niños y las niñas en los centros de estudiantes. De esta manera las escuelas que han acogido más población inmigrada en los últimos años están desarrollando una suerte de función "amortiguadora" al emerger como espacios de cuidado y de acogida especiales dentro de un clima adverso de instalación social. El hecho que desarrollen espacios y actividades extra-programáticas con las familias y niños/as o también a nivel comunitario con entidades públicas, centros culturales u ONG de sus zonas geográficas ha permitido a su vez proveer a las familias migradas de una red social más amplia y posibilitar la articulación entre ellas.

"La idea es hacer extensión cultural y desde esa perspectiva nosotros queremos irradiar a la comunidad externa de la escuela, de tal modo de que en ese afán podamos involucrar a los comerciantes del sector, podamos involucrar a gente que es residente antigua desde hace mucho tiempo, porque todavía quedan y también son personas muy descontentas con lo que hay porque se sienten como invadidos, con los cité, con los migrantes, eso también son situaciones que... las juntas de vecino, aquí la 35 de Bellavista, la 34 de Patronato, también están involucradas en este proceso (...) eso también incide mucho en el cuidado y protección de la escuela y que la sientan como un espacio que efectivamente pertenece" (Entrevista Directivo).

En este proceso cobran relevancia muchos rumores sociales y estereotipos respecto de las comunidades extranjeras que se difunden fuera de la escuela, especialmente respecto de personas peruanas, colombianas y dominicanas. Entre éstas, en las entrevistas se destacan generalizaciones tales como que "copan los servicios públicos, especialmente las escuelas", "son bulliciosos y alegadores", "son sucios", "traen enfermedades", "ha aumentado la delincuencia y la venta de drogas en el barrio", "son de vida fácil”. Principalmente, en este aspecto se destacan características "culturales" que ciertos grupos extranjeros poseerían (por ejemplo, el carácter desinhibido de la población colombiana y dominicana) y también en las formas de ocupación del espacio público. En ello, se generalizan conductas a todo un colectivo, y por otro, se invisibilizan comportamientos individuales que escapan a esa misma generalización. De esta manera, estos discursos terminan "culturalizando" a los colectivos de inmigrados a partir de supuestas características comunes, afectando con estas categorizaciones la autoestima de los/las hijos/as que son los estudiantes de las escuelas. 
En los grupos de discusión de niños y niñas se destacaron diversas experiencias y hechos que sí apuntan a cierta aprensión, indiferencia y/o rechazo hacia ellos/as dentro de algunas escuelas, que pasan por diferentes grados: desde las burlas y bromas que son consideradas como "parte de la cultura chilena" y que en el caso de la infancia migrante se relaciona con su procedencia; hasta comentarios más despectivos, burlas más ofensivas, acosos más explícitos y actos de violencia directa de parte de sus pares; como también algunas diferenciaciones por nacionalidad, hechas por algunos/as profesores/as y directivos.

“Tenemos nuestras propias reglas, si molestai tení que aguantarte que te molesten nomás po, así somos en la sala y sin andar acusando a la profe" (Grupo discusión niños/as chilenos/as).

También se visualiza una cierta idea de normalización de la molestia: es decir, se considera como algo común o evidente para el grupo etario que existan relaciones de tensióndistensión, bromas y sarcasmos en diferentes grupos por edad, pero que esto les "pasaría a todos y todas", y no sólo a quienes provienen de otro país. De esta manera, se tiende a normalizar cierto tipo de comportamiento de rechazo o exclusión, lo que puede ser riesgoso para la convivencia escolar porque por un lado, invisibiliza la discriminación que pueda experimentar efectivamente una persona extranjera al igualar a toda la infancia y adolescencia al mismo estatus y las mismas prácticas que las nacionales, y además porque resta valor a las experiencias que sí son consideradas como negativas por parte del mismo alumnado.

"Sí, entre los chiquillos, pero no sale de la normalidad, no es porque hayan haitianos aumentan esas situaciones de discriminación. Siempre han estado, entonces que se hayan integrado ellos no provoca que esto se haya disparado. Se molestan, pero así como podrían molestarse entre chilenos. El color de la piel, o sea, ese es el mecanismo de hostigar. Seguramente tienen dificultades y la única manera de mostrar su molestia es indicando que son negros o algo parecido, es el mas fácil, porque echarle un garabato puede que no lo vayan a entender" (Entrevista Directivo).

Esto queda más claro a partir de lo señalado en uno de los grupos de discusión con niños/as extranjeros/as:

"E: Y cuando ustedes los molestan a los chicos ¿qué le dicen ellos?

N1: Yo pienso que ellos se van a la parte racismo.

$\mathrm{E}:$ ¿Y a qué te refieres con racismo?

N1: A mí me dicen Negro o Coffee.

E: ¿Y a ti eso te molesta?

N1: A mí también antes me decían así, pero me dejaron. Ahora me llama Peta.

N2: Mmm, más o menos. Me dicen cosas.

N3: A ella le dicen Zombie"

(Grupo de discusión niños/as migrantes).

Un tema que emerge también en los grupos de discusión es el énfasis culturalista que se le asigna a ciertos comportamientos: niños/as chilenos/as sienten que los otros y las otras “extranjeros/as" son diferentes por su cultura, por el país de donde vienen. También destacan 
que los niños y las niñas migrantes se juntan sólo con su mismo grupo de procedencia, y consideran que eso dificulta las relaciones con el resto de compañeros/as sean chilenos/ as o de otras nacionalidades. No se considera mayormente que este comportamiento de "encierro" en su propio colectivo pueda deberse a las burlas o las presiones que reciben por su nacionalidad, más bien, se enfatiza que se debe a que "ellos se comprenden mejor entre ellos".

E: ¿Cómo describirían a los niños y niñas extranjeras? ¿Porque en el curso son todos haitianos no?

$\mathrm{N}$ 1: Eh no, hay dos peruanos, que no vinieron hoy día.

E: ¿De qué te ríes?

N 1: Que el compañero lo molesta porque es peruano.

E: ¿Por qué te molestan?

$\mathrm{N}$ 1: Porque es como peruano, por el color de piel.

N 2: Le dicen anda pal río Mapocho.

E: ¿Ustedes como describen entonces a sus compañeros extranjeros?

$\mathrm{N}$ 1: No discrimino, pero hablan muy fuerte sí.

N 2: Gritan.

N 3: Y cuando dicen que se callen no se callan a veces. Y cuando gritan, gritan más fuerte

E: ¿Pero ustedes creen que eso es algo particular de sus compañeros haitianos?

Todos: Sí.

N 1: Pero siempre la tía, los retan a ellos también, porque hablan más fuerte."

(Grupo de discusión niños/as chilenos/as)

"N4: Hay un grupo, que no es por sonar racista, pero que son la mayoría extranjero, peruanos. Un grupo que se excluye del resto, ellos mismos como que se alejan.

N5: Yo antes estaba en el mismo colegio que una de ellas, y se excluía ella sola también.

E: ¿Ustedes nunca se han preguntado por qué hacen eso o tienen alguna teoría?

N1: Yo creo que quieren estar solas.

N2: Quizás creen que no las vamos a aceptar.

N3: Yo creo que como se juntan con las personas de su mismo país como que son peruanos y se sienten en la misma cultura.

N4: Hay personas que llegan y no se sienten bien con el curso o con ellos mismos.

N5: Puede que piensen ellos que nosotros como somos chilenos no los vamos a aceptar.

N3: Yo tengo la teoría de cómo se vienen a Chile, y lo más probable es que se devuelvan, yo creo que no se quieren encariñar con nadie y se quieren devolver a su país lo más pronto posible"

Grupo de discusión niños/as chilenos/as)

En las experiencias narradas desde la infancia migrante y chilena, cobra especial relevancia el tema de la "negritud" o la discriminación que pueden cruzar los niños y las 
niñas por rasgos fenotípicos, lo que afecta principalmente a afrodescendientes colombianos y a la comunidad haitiana. De esta manera, las niñas colombianas y haitianas suelen declarar más experiencias de acoso o de burlas que otros niños, y sus relatos apuntan a una suerte de hipersexualización de sus cuerpos.

"N3: Porque son pesados, porque son desordenados. Nuestros padres nos dicen que no nos juntemos con personas desordenadas y que dicen garabatos y que no respetan a los profesores y preferimos alejarnos para no decir garabatos.

N1: Sí, nos dicen cosas feas.

N2: Dicen negra devuélvete a tu país, te voy a matar con un balazo.

N2: Sí, son pesados. Dicen "negro cochino, anda a tu casa".

E: ¿Y ustedes qué hacen con eso?

N1: Nada, los ignoramos.

E: ¿Y a ustedes las respetan?

N2: Un poquito, más o menos.

E: ¿Por qué más o menos?

N1: Porque se acostumbraron a nosotras.

N2: Dicen "oye negra, ven acá".

E: ¿y ustedes les molesta esto?

N2: No, no me molesta, porque ya nos acostumbramos.

E: ¿Pero al principio les molestaba?

N1: Sí.

E: ¿Y ustedes le dijeron a la profesora o al director que les molestaba?

N1: Yo sí, al inspector.

N2: Sí, y le dicen "niños, no tienen que hacer eso, porque son de otro país, tienen que tratarlos bien".

E: ¿Y el inspector qué les dijo?

N1: Nos dice que aprendamos el lenguaje de ellos, que tenemos que llevarnos bien con ellos."

(Grupo de discusión niños/as migrantes)

“E: ¿Parece más chilena que peruana? ¿Por qué?

N1: Porque es blanquita.

N2: Por el color de piel.

N3: No dice pe, dice pues.

E: ¿Y tú te juntas con compañeros de otro curso?

N1: Sí, más grande.

N2: Todo el colegio le pega a él, no se hace respetar.

E: ¿Y por qué te molestan tanto?

N1: Por su idioma.

(Grupo de discusión niños/as migrantes).

"N 1: hay que entran de otros cursos... Y a veces las tratan mal (a sus compañeras haitianas) y nosotros las decimos que paren, que dejen de molestarlas... a los hombres. E: ¿Cómo... las tratan mal?

$\mathrm{N}$ 1: Les dicen negras tal por cual y nosotros igual las defendemos un poco. 
E: ¿Qué hacen ustedes?

N 1 y 2: Los echamos pa' fuera"

(Grupo de discusión niños/as chilenos/as).

Muchas veces estas actitudes de aprensión menor hasta el rechazo más explícito, no sólo afecta el rendimiento educativo de niños y niñas, sino que también genera niveles de angustia, que pocas veces es tratada ante la falta de recursos de los mismos establecimientos. En algunas escuelas se ha llegado a violencia directa con población extranjera. Por ello, desarrollan algunas actuaciones o intervenciones puntuales, pero no se evidencian protocolos y programas explícitos de combate al bullying escolar:

"En séptimo y octavo existe más dificultades entre chilenas y haitianas, no es tan intensa, son episodios donde han tenido que intervenir...Nos juntamos con los papás, y la asistente social, el inspector general y la orientadora, porque han sido en general episodios de violencia grandotes, donde hemos visto enfrentamiento verbal y físico" (Entrevista Directivo).

Por otro lado, algunos alumnos/as enfatizan que ciertos profesores/as hacen distinciones entre chilenos/as y extranjeros de acuerdo al rendimiento que tienen en sus ramos, y eso les molesta y provoca más la posibilidad de "molestar" a sus compañeros/as extranjeros/as, especialmente si son mujeres.

Niños y niñas destacan también que a veces emerge la diferencia entre chilenos/ extranjeros en las formas de llamarles la atención de algunos profesores/as. Esto se evidencia en un caso en que una determinada profesora se dirigió con un trato distinto a los/as niños/as migrantes, generando molestia en todo el grupo de niños/as (chilenos/as y extranjeros), situación que más adelante en el curso fue abordada en orientación.

"N4: Sí, él estaba de espaldas y la profe estaba hablando y no le estaba poniendo atención. Y viene la profesora y dice "esa comunidad peruana que habla, mejor que se vayan".

N5: Pero yo creo que lo dijo en buena onda.

N2: Yo creo pero no debía tratarnos así como comunidad peruana, sino que también decirles por su nombre.

E: ¿Y a ustedes les molesto eso?

N2: Nos amargamos y dijimos "pero estamos copiando" y dijo "no, no, no están copiando".

N5: fue hace dos años po".

(Grupo de discusión niños/as migrantes).

N3: O sea, sí, una vez la profe de lenguaje, no sé si lo hizo de broma, pero ella estaba de vuelta escribiendo con una compañera, y la profe va y le dice "te voy a llevarte a tu país, peruana" una cosa así.

N4: "Devuélvete a tu país".

N3: Sí, cosas así le dijo la profesora. Entonces es que ellos se deben sentir mal con los profes que le digan eso que los alumnos, porque son profesores y son mayores de edad.

(Grupo de discusión niños/as chilenos/as). 
En las escuelas también se identificaron prácticas de diferenciación cultural en que se ubica al/la niño/a como un "otro culturalmente distinto" en comparación a los/as niños/ as nacionales, estableciendo una cultura que opera como parámetro de los conocimientos sociales e históricos que los/as niños/as debiesen portar. Esto genera formas de diferenciación que ponen al niño/a migrante en un lugar de desconocimiento y de saber desigual, en la que a su vez, la diferencia cultural es reforzada por medio de estigmatizaciones generalizadas dadas su condición de extranjería.

Algunos miembros directivos y docentes destacan las diferencias culturales entre países como un factor que puede causar problemas en las relaciones internas dentro de las escuelas: se mencionan casos sobre todo respecto de las relaciones entre niños y niñas y los roles de género que se practican en diferentes contextos. En ese sentido, hay algunos micro-machismos y comportamientos poco adecuados al marco escolar de ciertos grupos concretos -se enuncia a haitianos y peruanos-, pero a la vez, no se considera críticamente de la misma manera al grupo nacional (en otras palabras, se considera que estos niños por su "cultura" se comportan de esta manera, pero no se visualiza que también comportamientos micromachistas similares se gestan entre niños/as nacionales).

"A veces hemos tenido el problema con el tema de que ellos piropean a las chiquillas y a ellas les moleta y a veces a las chilenas no les gusta, pero entre ellos es distinto, entonces hemos tenido apoderados que dicen que "este niño haitiano anda molestando a mi chiquilla”, le toco el trasero, cosas así, que los de acá no lo hacen porque saben que socialmente no es aceptable, e cambio hay algunos haitianos que esa parte no la manejan, porque -según el facilitador lingüísticoallá es distinto" (Entrevista Directivo).

"Lo único es que algunos colegas como estamos acostumbrados al silencio, el tono de voz, es que yo le dije a la otra profe, "es su forma de ser, no son igual que nosotros" (Entrevista Docente).

¿Qué hacen las escuelas para intervenir las actitudes más discriminatorias hacia niños/ niñas migrantes? Algunas desarrollan actuaciones reactivas, y otras tienen dentro de los reglamentos de convivencia protocolos para atender y detener este tipo de eventos. No obstante, son de carácter generalista y no específico vinculado al bullying por procedencia nacional. Uno de los esfuerzos es visibilizar el aporte que a juicio de los/as entrevistados/ as, aporta la presencia de las personas migrantes a la escuela. Esta valoración positiva se considera en diversos niveles: aporte de la diversidad cultural; aporte lingüístico; aporte a la diversidad fenotípica; aporte en formas educativas y valores.

\section{CONCLUSIÓN}

La reflexión en torno a los factores de inclusión educativa hoy es fundamental para Chile y su sistema escolar, en especial si se considera que la población extranjera en las escuelas es parte de una realidad que debe ser visibilizada para asegurar el derecho a la educación para todos y todas. Y esta reflexión debe ser lo suficientemente amplia para identificar todos los factores que en la institución educativa determinan la participación de niños y niñas y su 
acceso al aprendizaje. Al respecto, desde la información obtenida en este estudio se puede afirmar que debido a las normas existentes en Chile el acceso al sistema educacional está garantizado, sin embargo, la situación tiende a ser más compleja para la población que carece de documentos del país de origen. Es cierto que no se niega matrícula a niños y niñas en condición irregular, pero no se puede desconocer que la asignación de un RUT provisorio dificulta otros procesos que se desarrollan dentro del sistema escolar, tales como el acceso a becas de alimentación, certificación de estudios, participación en actividades, etc.

A eso se suma que son las autoridades locales e incluso los directivos de las escuelas quienes toman decisiones en torno a los beneficios que pueden llegar a niños y niñas, lo que denota un cierto margen de arbitrariedad que por cierto en los casos estudiados ha jugado a favor de la inclusión, pero que por sobre todo debiera ser parte de una lógica institucional. Esto pone en evidencia la importancia del rol de los funcionarios públicos en la provisión de servicios a las familias migrantes, al modo como es mostrado en los estudios de Lipsky $(1980,1996)$ y Dubois (2003).

Como se ha hecho evidente en otras investigaciones (Autor et al., 2015) en algunas escuelas debido a la dificultad administrativa que implica la presencia de niños y niñas en condición irregular, se opta por derivarlos a otros centros, lo que en la práctica corresponde a la negación de la matrícula y la vulneración de un derecho.

Los factores pedagógicos que favorecen el proceso de inclusión también son relevantes, toda vez que la carga cultural que traen niños y niñas muchas veces se transforma en un factor que dificulta el acceso. El caso más significativo corresponde a niños y niñas haitianos que no hablan castellano y frente a lo cual las escuelas no cuenta con herramientas específicas.

La barrera idiomática opera como un factor transversal que impacta en el acceso al sistema educativo y las relaciones entre pares. Como se indicó antes, las escuelas no cuentan con docentes capacitados para hacerse cargo de esto, aunque sí con algunos apoyos por parte de los municipios (es el caso de la municipalidad de Quilicura que cuenta con un mediador intercultural que visita las escuelas).

Por último, en cuanto a las relaciones que se dan al interior de las escuelas estudiadas, aparecen algunos hechos de interés: aunque no existen grandes conflictos entre estudiantes por motivos de nacionalidad, se observa que por lo general los grupos no se relacionan entre sí, lo cual se hace mucho más fuerte a medida que aumentan en edad. Más concretamente, lo que relatan los agentes educativos es que por ejemplo los niños chilenos y extranjeros/ as más pequeños/as no tienen problemas en vincularse entre sí, sin embargo, a medida que crecen comienzan a relacionarse casi exclusivamente con sus connacionales. Esto es más evidente para quienes se encuentran en octavo año y mucho más claro en el liceo de secundaria estudiado. Como sea, lo relevante es que el fortalecimiento del grupo de nacionales no favorece los procesos de inclusión.

Por otro lado, ocurren situaciones en las escuelas que no son inmediatamente asumidas como formas de discriminación o bullying, lo que se explica porque han sido internalizadas como algo que es parte de la vida cotidiana del espacio escolar. Quienes se ven afectados en mayor medida por este tipo de situaciones son los niños y niñas afro descendientes, sobre quienes también recae, en especial en el caso de las mujeres, una cierta "hipersexualización" que deriva en estereotipos y otras formas de burla. Por su parte, la relación con el mundo adulto tampoco está exenta de dificultades. Si bien se mencionan casos puntuales y no generalizables, no deja de ser llamativo que algunos docentes utilicen 
expresiones discriminatorias para llamar al orden a sus estudiantes o para referirse a ellos en los casos en que se provocan situaciones que alteran el normal desarrollo de las clases.

Un ámbito que no puede dejar de ser mencionado es lo relativo a las políticas educativas propias de las escuelas. Si bien la mayoría de los casos estudiados se reconocen como instituciones inclusivas, muchas veces lo hacen por una suerte de asociación con los programas de integración de apoyo a niños y niñas con necesidades educativas especiales y no necesariamente rescatando el valor de la multiculturalidad. Dicho de otra manera, todos los centros educativos tienen conciencia de la presencia de niños y niñas extranjeras, pero no todos asumen en forma activa lo que significa la multiculturalidad que viven a diario. $\mathrm{Y}$ en ese sentido no es lo mismo que una escuela se declara inclusiva a que reconozca abiertamente la diversidad de origen de sus estudiantes como un valor.

Es innegable que Chile y el sistema educativo han avanzado en la generación de vías para la inclusión, sin embargo, es un desafío asumido en el espacio escolar de manera reactiva y en especial en las comunas donde se han asentado las personas inmigradas. No se puede desconocer que aún restan desafíos importantes por asumir y acciones que realizar para garantizar una efectiva inclusión de la infancia inmigrante. Generar estrategias que permitan superar los nudos críticos identificados es relevante en el contexto actual, en que todo indica que los procesos migratorios se incrementarán en el tiempo. Hacerlo no solo permitirá resolver cuestiones de tipo práctica, las que por cierto son importantes, sino también transitar hacia la profundización democrática y la generación de mayores niveles de equidad y justicia social para todos y todas.

\section{REFERENCIAS BIBLIOGRÁFICAS}

Autor (2009). Educación Intercultural en la escuela de hoy: reformas y desafíos para su implementación. Revista Latinoamericana de Educación Inclusiva, 3 (2), 181-200.

Autores (2007). La identidad en la encrucijada: migración peruana y educación en el Chile de hoy. II Congreso Internacional de Etnografía y Educación, Migraciones y ciudadanía. EMIGRA. Barcelona: Universidad Autónoma de Barcelona. Recuperado el 30 de noviembre de 2016 desde https://ddd.uab.cat/pub/emigrawp/emigrawp_a2007n3/emigrawp_a2007n3p1.pdf

Autores (2015). Inmigrantes en Chile: oferta programática, participación, inclusión y vulnerabilidad. Informe Final de Investigación realizada para el Ministerio de Desarrollo Social. Santiago: MIDESO. Documento no publicado.

Blanco, R. (1999). Boletin del Proyecto Principal de Educación para América Latina y el Caribe, 48, 55-72. Santiago: UNESCO.

Booth, T. y Ainscow, M. (2000). Guía para la evaluación y mejora de la ecuación inclusiva. Index for inclusión. Madrid: Consorcio Universitario para la Educación Inclusiva.

Carrasco, S. (1998). Interculturalitat I Educació. Aportacions per a un debat entre l"antropologia social y la pedagogía. Barcelona: Universidad Autónoma de Barcelona.

Chiodi, F. y Bahamondes, M. (2001). Una Escuela, Diferentes Culturas. Temuco: Corporación Nacional de Desarrollo Indígena.

Departamento de Extranjería y Migración del Ministerio del Interior y Seguridad Pública (2015). Migración en Chile 2005-2014. Santiago: DEM.

Dubois, V. (2003). La sociologie de l'action publique.De la socio-histoire à l'observation des pratiques (et vice-versa). En Laborier, P. y Trom, D. (Ed.), Historicités de l'action Publique. (pp. 347-364). Paris: PUF. Recuperado el 25 de mayo de 2015 desde http://halshs.archivesouvertesr/docs/00/46/43/22/PDF/sociologiedelalactionpbliq.pdf 
Echeita, G. (2008). Inclusión y Exclusión Educativa. Voz y Quebranto. Revista Electrónica Iberoamericana sobre Calidad, Eficacia y Cambio en Educación, 6 (2), 9-18.

Fernández, M. y Terrén, E. (2008). De inmigrantes a minorías: temas y problemas de la multiculturalidad. Revista de Educación, 345, 15-21.

García, A. Sáez, J. (1998). Del racismo a la interculturalidad. Madrid: Narcea.

Leiva, J. (2007). Interculturalidad, gestión de la convivencia y diversidad cultural en la escuela: un estudio de las actitudes del profesorado. Revista Iberoamericana de Educación, 46 (2), 1-14.

Lipsky, M. (1980). Street Level Bureeaucracy: Dilemnas of the Individual in Public Services. New York: Russel Sage Fundation.

Lipsky, M. (1996). Los empleados de base en la elaboración de políticas públicas. En Brugué, Q. y Subirats, J. (Ed.), Lecturas de Gestión Pública (pp. 279-298). Madrid: INAP.

López, L. (1997). La diversidad étnica, cultural y lingüística latinoamericana y los recursos humanos que la educación requiere. Revista Iberoamericana de Educación, 13, 47-98.

Martínez, J. (2003). El encanto de los datos. Sociodemografía de la inmigración en Chile según el Censo de 2002. Santiago: Comisión Económica para América Latina y el Caribe (CEPAL).

Martínez, J. (2011). Migración Internacional en América Latina y el Caribe. Nuevas tendencias, nuevos enfoques. Santiago: Comisión Económica para América Latina y el Caribe (CEPAL).

Ministerio de Desarrollo Social (2014). Resultados Encuesta CASEN 2013. Santiago: MIDESO.

Ministerio de Desarrollo Social (2016). Resultados Encuesta CASEN 2014. Santiago: MIDESO.

Ministerio de Educación de Chile (2005). Ordinario Nº7/1008 (1531). Santiago: MINEDUC.

Ministerio de Educación de Chile (2015). Ley de Inclusión Educativa. Documento de Trabajo. Santiago: MINEDUC.

Ministerio de Educación de Chile (2016). Ordinario Nº2/000894. Santiago, MINEDUC.

Ogbu, J. (1991) Immigrant and Involuntary Minorities in Comparative Perspective. En Gibson, M. and Ogbu, J. (Ed.), Minority Status and Schooling. A comparative study of immigrant and involuntary minorities (pp.3-33). New York: Garland Publishing.

Pavez, I. (2012) Inmigración en Chile. Experiencias de la niñez peruana en Santiago de Chile. Revista de Estudios Tranfronterizos, 12 (1), 75-99.

Ruíz Olabuenaga, J.I. (1996) Metodología de la Investigación Cualitativa. Bilbao: Universidad de Deusto.

Stefoni, C. (2003) Inmigración en Chile: una oportunidad a la integración. Santiago: Editorial Universitaria.

Subirats, J. (2010) Ciudadanía e Inclusión Social. Madrid: Fundación Esplai.

Torres, O. y Garcés, A. (2013) Representaciones sociales de migrantes peruanos sobre su proceso de integración en la ciudad de Santiago de Chile. Polis, 12 (35), 309-334. Recuperado el 30 de mayo de 2015 desde http://www.scielo.cl/scielo.php?script=sci_arttext\&pid=S071865682013000200014\&lng=es\&tlng=es. 10.4067/S0718-65682013000200014.

UNESCO (2005) Guidelines for inclusion: Ensuring Access to Education for All. París:_UNESCO.

Unicef (2004). Convivencia y discriminación en el ámbito escolar. Santiago: Unicef.

Unicef (2011). Convivencia y discriminación en el ámbito escolar. Santiago: Unicef. 
